\title{
Preoperative transcranial Doppler and cerebral oximetry as predictors of delirium following valvular heart surgery: a case-control study
}

\author{
Sarah Soh ${ }^{1,2,3} \cdot$ Jae-Kwang Shim ${ }^{1,2,3} \cdot$ Jong-Wook Song $^{1,2,3} \cdot$ Nakcheoul Choi $^{1} \cdot$ Young-Lan Kwak $^{1,2,3}$ (])
}

Received: 30 July 2019 / Accepted: 29 August 2019 / Published online: 3 September 2019

C) Springer Nature B.V. 2019

\begin{abstract}
Delirium is a frequent and serious complication after cardiac surgery with cerebral hypoperfusion as one from the key pathophysiological mechanisms. Middle cerebral artery (MCA) mean blood flow velocity (MFV) measured by transcranial Doppler has been used as a marker of cerebral perfusion, and cerebral oximetry $\left(\mathrm{rSO}_{2}\right)$ value as a marker of its adequacy. This prospective observational trial examined the predictive value of MCA MFV and $\mathrm{rSO}_{2}$, measured immediately before induction of anesthesia, for delirium after valvular heart surgery in elderly patients. In 113 patients, delirium was evaluated for 7 days postoperatively, using the confusion assessment method for the intensive care unit. The primary endpoint was the occurrence of postoperative delirium. Overall, 16 patients (14\%) exhibited delirium. MCA MVF values could not predict the development of delirium. Preoperative statin use, geriatric depression scale score, and low preoperative $\mathrm{rSO}_{2}(<60 \%)$ showed association with delirium occurrence in univariable analysis. After multivariable analysis, only the low preoperative $\mathrm{rSO}_{2}(<60 \%)(\mathrm{OR} 6.748,95 \% \mathrm{CI} 1.647-27.652, P=0.008)$ remained as an independent predictor of delirium. Preoperative MCA MFV was not significantly associated with delirium after valvular heart surgery in elderly patients, while a low baseline $\mathrm{rSO}_{2}$ value was associated with a sevenfold increased risk of delirium.
\end{abstract}

Keywords Cardiac surgical procedures · Delirium · Heart valve diseases · Spectroscopy, Near-infrared · Ultrasonography, Doppler, Transcranial

\section{Introduction}

Delirium is recognized as a common postoperative complication of cardiac surgery, with serious consequences including morbidity, long-term cognitive dysfunction, and even mortality [1]. Therefore, proper assessment of risk factors related to delirium is of high priority.

Several studies have shed light on risk factors related to delirium following cardiac surgery, which include patient-,

Young-Lan Kwak

ylkwak@yuhs.ac

1 Department of Anesthesiology and Pain Medicine, Yonsei University College of Medicine, 50 Yonsei-ro, Seodaemun-gu, Seoul 03722, Republic of Korea

2 Yonsei Cardiovascular Hospital, Yonsei University College of Medicine, 50 Yonsei-ro, Seodaemun-gu, Seoul 03722, Republic of Korea

3 Anesthesia and Pain Research Institute, Yonsei University College of Medicine, 50 Yonsei-ro, Seodaemun-gu, Seoul 03722, Republic of Korea surgery-, and cardiopulmonary bypass (CPB)-related factors that converge to indicate the importance of cerebral hypoperfusion and systemic inflammation [2, 3]. Likewise, a decrease in cerebral blood flow, and subsequent conversion to anaerobic glycolysis, has been demonstrated in delirium [4]. Measurements of middle cerebral artery (MCA) mean flow velocity (MFV) by transcranial Doppler (TCD) in nonsurgical patients directly confirmed impairment of cerebral perfusion and showed correlations with cognitive dysfunction and delirium $[5,6]$.

Cerebral blood flow can be assessed non-invasively by TCD and indirectly by cerebral oximetry $\left(\mathrm{rSO}_{2}\right)$. Both methods have been applied during cardiac surgery to predict postoperative neurologic complications, with conflicting results $[7,8]$. Moreover, studies revealed impracticability of TCD as a continuous monitor [9]. Likewise, calculating the cumulative area or time under an arbitrary cut-off value of $\mathrm{rSO}_{2}$ during the entire surgery to predict neurocognitive outcome seems to be of limited clinical significance [10]. In contrast, baseline MCA MFV or $\mathrm{rSO}_{2}$ values may represent the patient's individual reserve against cerebral hypoperfusion 
and offer advantage in terms of clinical practicability when compared to continuous monitoring [11, 12]. Indeed, a previous study in cardiac surgery has shown a close relationship between low baseline MCA MFV and postoperative cognitive decline [11]; however, evidence regarding the correlation with delirium is lacking.

In the current observational trial, we aimed to assess the predictive value of preoperative $\mathrm{MCA} \mathrm{MFV}$ and $\mathrm{rSO}_{2}$ value for the occurrence of delirium in elderly patients undergoing valvular heart surgery.

\section{Materials and methods}

\subsection{Patients}

This prospective observational study was sanctioned by the Institutional Review Board (4-2015-0319) of the Yonsei University Health System, Seoul, Republic of Korea, and registered at www.ClinicalTrials.gov (identifier: NCT02478736). Informed consent was obtained from all individual participants included in the study. The following methods were carried out in accordance with the relevant regulations. The study included 113 patients, who were enrolled from June 2015 to March 2017. The inclusion criteria were: (1) age $\geq 60$ years, (2) scheduled for valvular heart surgery. The exclusion criteria were: (1) inability to undertake the mini-mental state examination (MMSE) and Confusion Assessment Method for the intensive care unit (CAM-ICU) procedures, (2) patients with unstable hemodynamics or who were intubated before surgery, (3) diagnosis of stroke, transient ischemic attack or neurologic diseases, and (4) patients diagnosed with significant ( $\geq 50 \%)$ intracranial stenosis confirmed by angiography.

\subsection{Study endpoints and power calculation}

The endpoint for this study was the occurrence of delirium. The primary outcome measures were the predictive values of MCA MVF and $\mathrm{rSO}_{2}$ values for delirium following valvular heart surgery in elderly patients. A sample size calculation was performed under the assumption that MCA MFV could predict the occurrence of delirium with similar efficacy as $\mathrm{rSO}_{2}$ values reported in a previous study [12]. Thus, the area under the receiver operating characteristic curve (AUROC) for MCA MVF was assumed to be 0.7. Because the expected incidence of delirium in a similar subset of surgical patients was approximately $20 \%$ (institutional data), to detect a 0.2 difference in the AUROC at a 2-sided alpha $=0.05$ with $80 \%$ power, 105 participants were required. Anticipating a dropout rate of $40 \%$ due to the high incidence of poor acoustic temporal window in elderly Asian female participants, 175 patients were enrolled.

\subsection{Neurologic assessment}

Trained research personnel not involved in the study examined all patients for delirium daily, first using the Richmond Agitation Sedation Scale to examine the level of consciousness, then using the Korean CAM-ICU [13] or CAM to detect delirium. A positive CAM-ICU/CAM test result during the first 7 days postoperatively was defined as delirium.

Before anesthetic induction, all patients received a TCD evaluation by the same trained researcher [bilateral MCA blood flow velocity monitoring using power M-mode Doppler (TCD 100 M, Spencer Technologies Inc., Seattle, WA)]. Transtemporal insonation was performed with a $2 \mathrm{MHz}$ pulsed TCD probe, and data on peak systolic velocity (PSV) and end-diastolic velocity (EDV) in the bilateral MCA were collected. MFV and pulsatility indices $(\mathrm{PI})[\mathrm{PI}=(\mathrm{PSV}-\mathrm{EDV}) \div \mathrm{MFV}]$ provided by automatic calculation. PSV and EDV were measured three times on each side at $2-3$ min time intervals, and the mean values of MFV and PI were used for analysis. If the measured MCA MFV exceeded $100 \mathrm{~cm} / \mathrm{s}$, the patient was dropped from the study, because significant MCA stenosis could not be ruled out.

In addition, the patients underwent continuous bihemispheric $\mathrm{rSO}_{2}$ observation. The $\mathrm{rSO}_{2}$ was measured using the INVOS ${ }^{\mathrm{TM}}$ Cerebral/Somatic Oximeter 5100 (Somanetics, Troy, MI, USA) with bihemispheric Near-Infrared Spectroscopy sensors, and the mean of the values from both sides was used for analysis. The baseline value of $\mathrm{rSO}_{2}$ was obtained in the supine position in room air before anesthesia, and measurement was continued until ICU transfer. The lowest and highest intraoperative $\mathrm{rSO}_{2}$ values were also obtained.

\subsection{Study design and perioperative measurements}

The patients were evaluated with the CAM-ICU a day before surgery to assess their eligibility for this study. Likewise, MMSE and geriatric depression scale short form (SGDS) Korean Version [14] were also tested to assess baseline cognitive function and depressive mood. The patient characteristics, logistic EuroSCORE, co-morbid diseases, and chronic medications were documented. Laboratory tests were conducted for levels of hematocrit, albumin, leukocytes, c-reactive protein, creatinine, and estimated glomerular filtration rate. Intraoperative variables included type of surgery, durations of aortic cross clamp and CPB, and transfusion requirement. Postoperative evaluation included transfusion requirement, lengths of ICU and hospital stay, and the incidence of composite 
morbidity endpoints defined by the Society of Thoracic Surgeons, including acute kidney injury, prolonged ventilator care $(>24 \mathrm{~h})$, permanent stroke, hemostatic reoperation, deep sternal wound infection, and in-hospital mortality. Intraoperative hemodynamic parameters and laboratory variables, including the mean arterial pressure (MAP), cardiac index, $\mathrm{PaO}_{2}, \mathrm{PaCO}_{2}$, and hematocrit levels, were recorded at the following predetermined time points: (1) prior to anesthetic induction, (2) $10 \mathrm{~min}$ postinduction, (3) $10 \mathrm{~min}$ after CPB initiation, (4-6) 40, 80, and $120 \mathrm{~min}$ after CPB initiation, respectively, (7) $10 \mathrm{~min}$ after CPB-off, and (8) $5 \mathrm{~min}$ after sternum closure.

The predictors including results of TCD, $\mathrm{rSO}_{2}$, and perioperative variables were blinded to participants and trained personnel who examine participants for postoperative delirium to prevent additional information bias.

\subsection{Perioperative management}

Perioperative care, including CPB management, was done according to an institutional standardized protocol. In brief, anesthesia was initiated using midazolam $(0.05 \mathrm{mg} /$ $\mathrm{kg}$ ) and sufentanil $(1.5 \mu \mathrm{g} / \mathrm{kg})$, and maintained with sufentanil $(0.5 \mu \mathrm{g} / \mathrm{kg} / \mathrm{h})$ and sevoflurane. Anesthetic depth was maintained at a score of 40-60 on the bispectral index (A-200 Bispectral Index ${ }^{\circledR}$ score monitor, Aspect Medical System Inc., Newton, MA). During the operation, MAP was maintained at $60-80 \mathrm{mmHg}$ using norepinephrine first, and if the target MAP could not be maintained with escalating doses of norepinephrine (maximum of $0.3 \mu \mathrm{g} /$ $\mathrm{kg} / \mathrm{min}$ ), vasopressin was added (2.4-4 unit/h). Milrinone was used in cases of left ventricular ejection fraction $<30 \%$, right ventricular dysfunction, or pulmonary hypertension. During CPB, the non-pulsatile flow rate was maintained at $2.0-2.51 / \mathrm{min} / \mathrm{m}^{2}$ using a tepid temperature $\left(32-33{ }^{\circ} \mathrm{C}\right)$ and alpha-stat management. Packed red blood cells were transfused when the hematocrit was $<20 \%$ during CPB or $<25 \%$ otherwise. Fresh frozen plasma (FFP) and platelets were transfused at the discretion of the attending anesthesiologist and cardiac surgeon during surgery. After surgery, if bleeding exceeded more than $200 \mathrm{ml} / \mathrm{h}$ for 2 consecutive hours, FFP or platelets or both were transfused, if the International Normalized Ratio was greater than 1.3 or the platelet count was less than $50 \times 10^{3} / \mathrm{ml}$.

During ICU care, postoperative sedation was achieved using propofol at 1 to $2 \mathrm{mg} / \mathrm{kg} / \mathrm{h}$ and remifentanil at $0.1 \mu \mathrm{g} / \mathrm{kg} / \mathrm{min}$, if required. Postoperative pain was assessed using a $10-\mathrm{cm}$ visual analogue scale $(0$, pain-free; 10 , worst imaginable pain) and controlled with fentanyl-based intravenous patient-controlled analgesia.

\subsection{Statistical analyses}

SPSS software for windows (version 23, SPSS Inc., Chicago, IL) was used for analyses. Data were expressed as the number of patients (percentage), mean \pm standard deviation (SD), or median (interquartile range, IQR). Continuous data were tested for normality and analyzed using the Mann-Whitney $U$ test or independent $t$ test, as required. Categorical data were analyzed using Fisher's exact test or the Chi square test. Intergroup comparisons of the serially assessed data were done using linear mixed models with an unstructured covariance matrix. Two fixed effects were included, the between-subjects factor of the occurrence of delirium and the within-subject factor of time.

Variables that yielded a $P$ value of less than 0.05 from the intergroup comparisons of perioperative data between patients with and without delirium were entered into the univariable logistic regression analysis. Variables with $P<0.05$ on that analysis were finally included in the multivariable analysis. A $P$ value less than 0.05 was deemed statistically significant, except for the serially assessed data, which was subjected to a Bonferroni correction. The optimal cutoff values for the continuous variables were determined by receiver operating characteristics (ROC) analysis.

\section{Results}

Data from 113 patients were included in the study (Fig. 1). Postoperative delirium was observed in 16 (14\%) patients within 7 days after surgery. The percentages of patients receiving statins, and the SGDS score, were significantly different between the groups (Table 1).

The MCA MFV and the percentage of patients with low preoperative MCA MFV $(<30 \mathrm{~cm} / \mathrm{s})$ were similar between the groups (Table 2). The preoperative $\mathrm{rSO}_{2}$ values $(56 \pm 6$ vs. $62 \pm 7, P=0.007)$ and intraoperative highest $\mathrm{rSO}_{2}$ values [67 (IQR 64-72) vs. 72 (IQR 68-78), $P=0.005$ ] were significantly lower in the delirium group than in the nondelirium group, but the lowest intraoperative $\mathrm{rSO}_{2}$ values were similar between the groups (Table 2).

Intraoperative hemodynamic and arterial blood gas analysis data were comparable between the groups over time. Also, the postoperative hemodynamic data and lowest hematocrit values were similar between the groups over time (Fig. 2).

The number of ICU days [3 (IQR 2-7) vs. 2 (IQR 1-3), $P=0.02]$ and number of hospital days [17 (IQR 12-27) vs. 10 (IQR 8-16), $P=0.002$ ] were increased in the delirium group than in the non-delirium group. The incidences of composite of morbidity endpoints ( $63 \%$ vs. $31 \%, P=0.014)$ and acute kidney injury ( $50 \%$ vs. $23 \%, P=0.032)$ were 
Fig. 1 Patient enrolment into the study

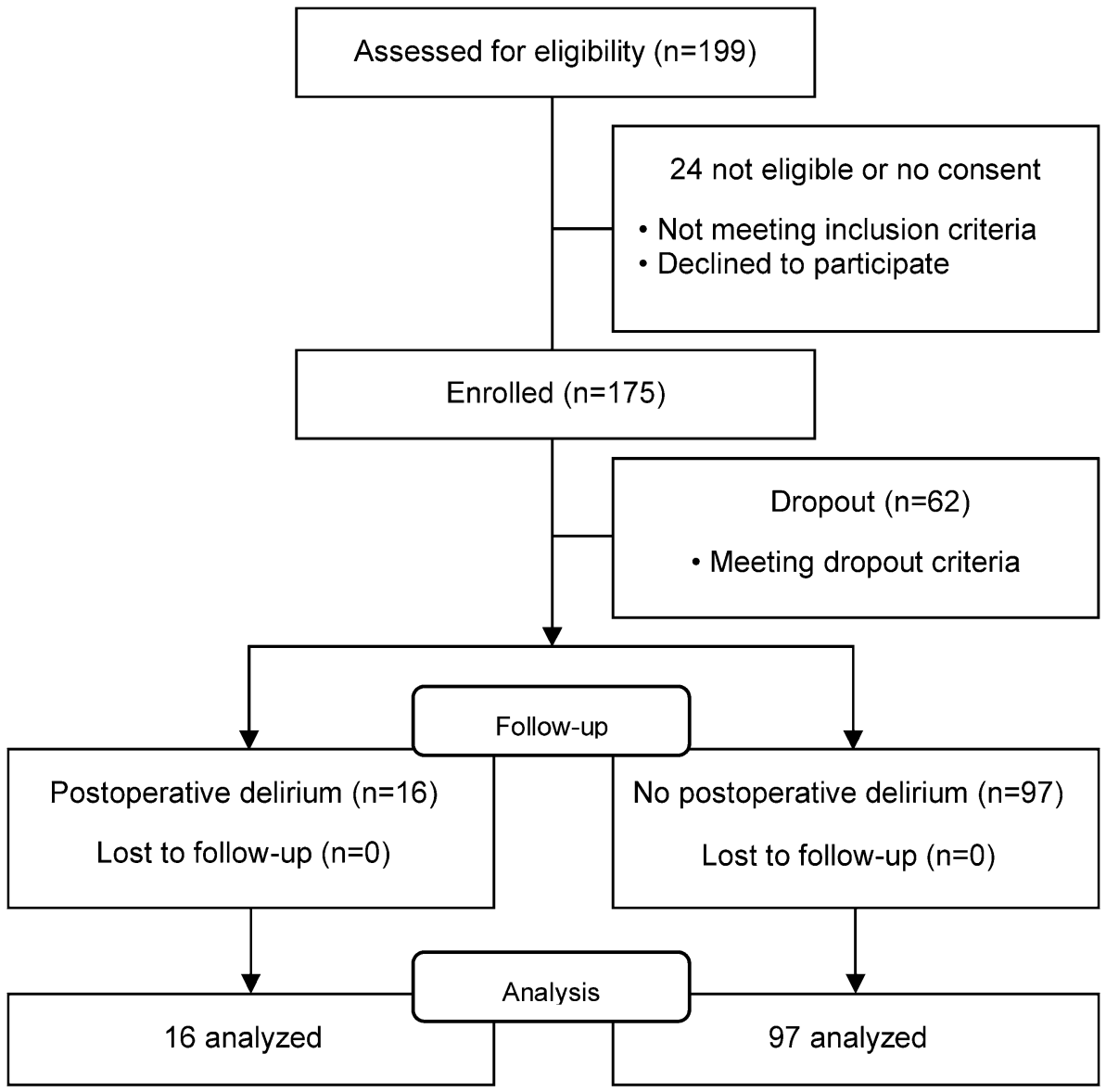

significantly higher in the delirium group than in the nondelirium group (Table 3 ).

In the multivariable analysis, only low baseline $\mathrm{rSO}_{2}$ $(<60 \%)$ [odds ratio (OR) 6.748 , 95\% confidence interval (CI) 1.647-27.652, $P=0.008]$ remained as an independent risk factor of delirium. Preoperative statin administration (OR 0.231, 95\% CI 0.046-1.155, $P=0.074$ ) and SGDS score (OR 1.194, 95\% CI 0.987-1.446, $P=0.069$ ) were associated with delirium, without statistical significance (Table 4).

The optimal cut-off value of preoperative $\mathrm{rSO}_{2}$ for predicting delirium after surgery was $59.5 \%$ (AUROC 0.739 , 95\% CI 0.611-0.866, $P=0.002$ ), yielding a sensitivity of $81 \%$ and specificity of $67 \%$ (Fig. 3).

\section{Discussion}

This prospective observational study found that the preoperative MCA MFV value assessed by TCD could not predict the development of delirium, whereas a low preoperative $\mathrm{rSO}_{2}$ value $(<60 \%)$ was linked to an estimated sevenfold increased occurrence of delirium.

Considering the clinical significance of delirium in cardiac surgery and the role of cerebral hypoperfusion in its pathogenesis $[15,16]$, a more thorough risk stratification involving the baseline reserve for cerebral perfusion may allow optimization of perioperative management. Preoperative $\mathrm{rSO}_{2}$ values, indirectly indicating the inadequacy of cerebral perfusion, have been shown to predict delirium after cardiac surgery, although they do not provide direct information on cerebral blood flow [12].

Cerebral blood flow velocity examined with TCD at the MCA has proven to be a valid tool of investigating cerebral perfusion [17]. Many studies exploring the relationship between cerebral hemodynamics measured with TCD and delirium or cognitive dysfunction have reported positive correlations [5, 6]. MCA MFV, PI, and the cerebral autoregulation index, measured by TCD, were found to be useful for diagnosing delirium superimposed on dementia [6], and for predicting sepsis-related delirium [18], although intraoperative TCD monitoring could not forecast delirium [7]. In cardiac surgical patients, TCD has been used mainly to investigate the association among hypoperfusion, microemboli, and postoperative cognitive dysfunction, yielding conflicting results $[19,20]$. Also, current anesthetic practice involving routine monitoring with bispectral index and cerebral oximetry in cardiac surgical patients limits the application of TCD as a continuous intraoperative monitor. 
Table 1 Baseline characteristics

\begin{tabular}{|c|c|c|c|}
\hline & Delirium $(n=16,14 \%)$ & Non-delirium $(n=97)$ & $P$ value \\
\hline Male, $\%$ & $11(69)$ & $60(62)$ & 0.597 \\
\hline Age, years & $71 \pm 5$ & $70 \pm 6$ & 0.674 \\
\hline Body surface area, $\mathrm{m}^{2}$ & $1.618 \pm 0.149$ & $1.663 \pm 0.154$ & 0.276 \\
\hline Educational level, years, IQR & $8(6-12)$ & $11(6-12)$ & 0.436 \\
\hline MMSE score $<24, \%$ & $3(19)$ & $13(14)$ & 0.699 \\
\hline Geriatric depression scale (short form) IQR & $4(1-7)$ & $2(1-4)$ & 0.029 \\
\hline EuroSCORE, IQR & $7(5-9)$ & $6(4-8)$ & 0.206 \\
\hline Diabetes mellitus, $\%$ & $3(19)$ & $27(28)$ & 0.553 \\
\hline Chronic kidney disease, $\%$ & $3(19)$ & $7(7)$ & 0.15 \\
\hline Pre-operative lung disease, $\%$ & $2(13)$ & $8(8)$ & 0.632 \\
\hline \multicolumn{4}{|l|}{ Pre-operative medication } \\
\hline Statin use, \% & $2(13)$ & $43(44)$ & 0.016 \\
\hline Beta blocker use, $\%$ & $6(38)$ & $29(30)$ & 0.567 \\
\hline Calcium channel blocker use, $\%$ & $1(6)$ & $23(24)$ & 0.186 \\
\hline RAS blocker use, $\%$ & $8(50)$ & $58(60)$ & 0.461 \\
\hline Diuretics use, $\%$ & $12(75)$ & $62(64)$ & 0.388 \\
\hline Pre-operative left ventricular ejection fraction, $\%$ & $57 \pm 13$ & $61 \pm 14$ & 0.27 \\
\hline Hematocrit, \% & $37 \pm 5$ & $38 \pm 6$ & 0.51 \\
\hline Albumin, g/dl, IQR & $3.9(3.6-4.3)$ & $4.1(3.7-4.3)$ & 0.476 \\
\hline Leukocytes, $/ \mu \mathrm{l}$ & $5805 \pm 1851$ & $5838 \pm 1664$ & 0.942 \\
\hline C-reactive protein, $\mathrm{mg} / \mathrm{l}, \mathrm{IQR}$ & $7.8(1.6-10.4)$ & $2.7(0.8-6.8)$ & 0.119 \\
\hline Creatinine, mg/dl & $1.02 \pm 0.25$ & $0.97 \pm 0.91$ & 0.8 \\
\hline
\end{tabular}

Data are presented as mean \pm standard deviation (SD), median (interquartile range, IQR), or number of patients (\%)

$I Q R$ interquartile range, MMSE the mini-mental state examination, $R A S$ renin-angiotensin system

Table 2 Pre-operative values of transcranial Doppler and perioperative values of cerebral oximetry

\begin{tabular}{llll}
\hline & Delirium $(\mathrm{n}=16)$ & Non-delirium $(\mathrm{n}=97)$ & $P$ value \\
\hline Mean cerebral blood flow velocity & & & \\
Left MCA, cm/s & $42 \pm 14$ & $44 \pm 14$ & 0.523 \\
Right MCA, cm/s & $42 \pm 15$ & $43 \pm 13$ & 0.738 \\
Lower MCA MFV, cm/s & $37 \pm 13$ & $39 \pm 11$ & 0.548 \\
Lower MCA MFV <30 cm/s, \% & $4(25)$ & $21(22)$ & 0.751 \\
Pulsatility index & & & \\
Left MCA, IQR & $0.85(0.75-1.06)$ & $0.89(0.76-1.03)$ & 0.924 \\
Right MCA, IQR & $0.92(0.82-1.04)$ & $0.88(0.76-1.02)$ & 0.413 \\
Low MVF combined with high PI, \% & $1(7)$ & $0(0)$ & 0.15 \\
Difference between left and right MCA & $7.7(4.7-12.7)$ & $8.5(3.3-20.1)$ & 0.531 \\
$\quad$ MFV, cm/s, IQR & & & \\
Pre-operative rSO,$\%$ & $56(6)$ & $62(7)$ & 0.007 \\
Lowest $\mathrm{rSO}_{2}, \%$, IQR & $43(37-46)$ & $45(38-49)$ & 0.267 \\
Highest $\mathrm{rSO}{ }_{2}, \%$, IQR & $67(64-72)$ & $72(68-78)$ & 0.005 \\
\hline
\end{tabular}

Data are presented as mean \pm standard deviation (SD), median (interquartile range, IQR), or number of patients (\%)

$M C A$ middle cerebral artery, $M F V$ mean blood flow velocity, $I Q R$ interquartile range, $r \mathrm{SO}_{2}$ cerebral oximetry

In the context of a single preoperative measurement, reduced MCA MFV was reported as an independent risk factor for short-term postoperative cognitive decline in cardiac surgical patients [11]. However, the connection between delirium and cognitive dysfunction remains elusive [21], and no comprehensive data exist regarding the predictive 

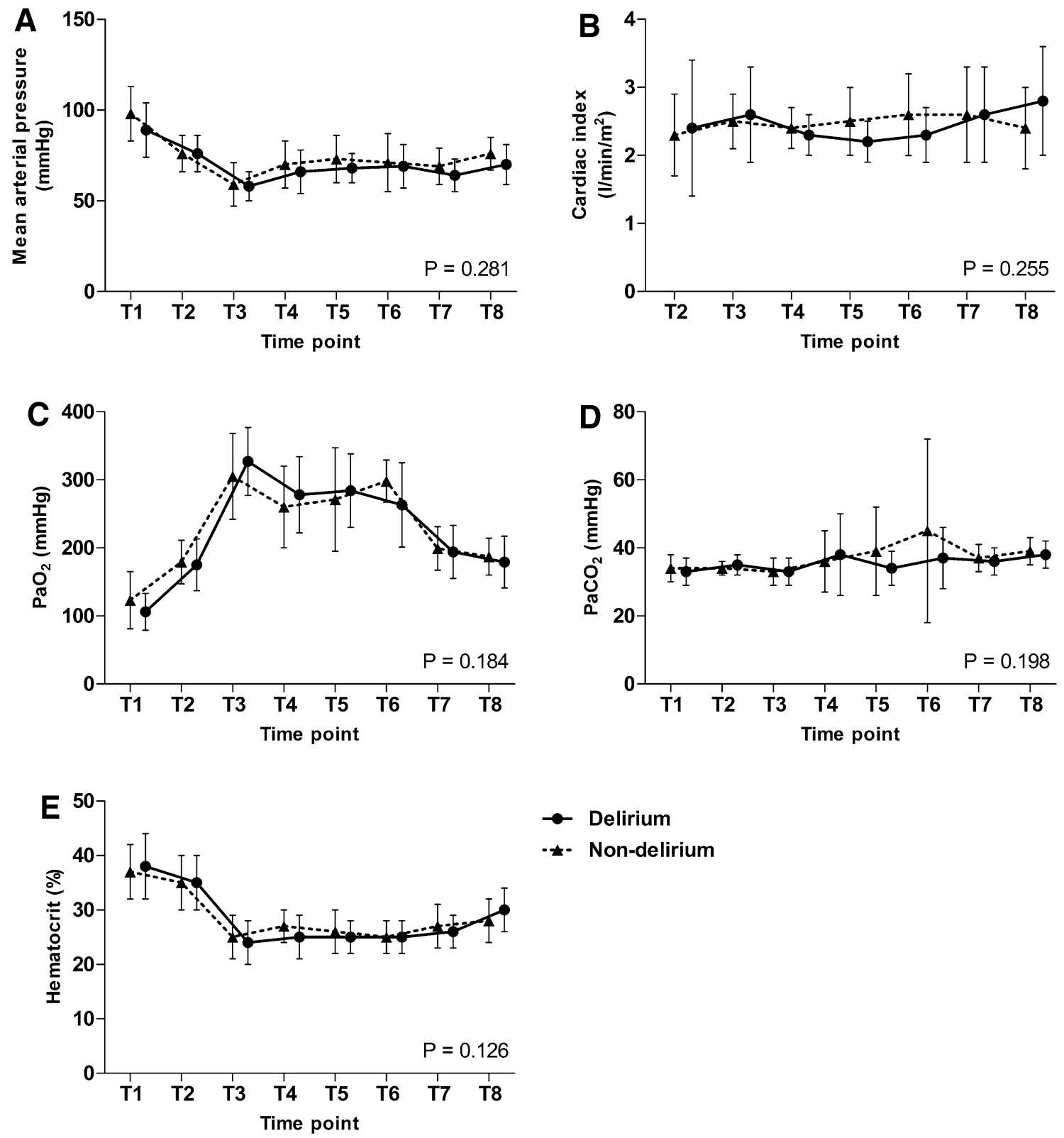

Fig. 2 Perioperative serial changes in hemodynamics and laboratory data. Serial changes in mean arterial pressure (a), cardiac index (b), the partial pressure of oxygen in the arterial blood (c), the partial pressure of $\mathrm{CO}_{2}$ in the arterial blood (d), and hematocrit levels (e) of patients with postoperative delirium (circle, solid line) compared with those without (triangle, dotted line). Variables were recorded at the following predetermined time points: (T1) prior to anesthetic

value of preoperative TCD values for delirium after cardiac surgery. We hypothesized that by providing direct information on cerebral blood flow and because it is able to identify patients with delirium in a non-surgical population, preoperative MCA MFV values could predict high risk for delirium in elderly patients with valvular heart disease. The type of surgery was limited to valvular heart surgery because most previous studies on delirium were focused on coronary induction, (T2) 10 min post-induction, (T3) $10 \mathrm{~min}$ after CPB initiation, (T4-6) 40, 80, and $120 \mathrm{~min}$ after CPB initiation, respectively, (T7) 10 min after CPB-off, and (T8) 5 min after sternum closure. The values of cardiac index were recorded at same time except timepoint (T1) before anesthetic induction. Error bars represent standard deviations

artery disease patients, while procedures other than isolated coronary artery bypass surgery were found to be independent predictors of delirium [22].

Contrary to our expectations, a significant relationship between the preoperative MCA MFV value and postoperative delirium could not be detected. One possible explanation is that the direct relationship between MFV and cerebral blood flow may be altered by reduced 
Table 3 Intraoperative and postoperative data
Table 4 Predictive power of chosen variables including pre-operative cerebral oximetry values for delirium according to logistic regression analyses

\begin{tabular}{llll}
\hline & Delirium $(\mathrm{n}=16)$ & Non-delirium $(\mathrm{n}=97)$ & $P$ value \\
\hline Surgical procedure, $\mathrm{n}(\%)$ & & & 0.13 \\
Mitral valve replacement/repair & $3(19)$ & $9(9)$ & \\
Aortic valve replacement/repair & $2(13)$ & $36(37)$ & \\
Tricuspid valve replacement/repair & $3(19)$ & $5(5)$ & \\
Multiple valve replacement/repair & $4(25)$ & $20(21)$ & \\
Valve replacement/repair +CABG & $1(6)$ & $10(10)$ & \\
Valve replacement/repair +other & $3(19)$ & $17(18)$ & 0.999 \\
Total circulatory arrest, n (\%) & $1(6)$ & $6(6)$ & 0.202 \\
Pre-operative cerebral perfusion pressure, & $63 \pm 11$ & $66 \pm 10$ & 0.125 \\
mmHg (MAP-CVP) & & & 0.28 \\
Duration of CPB, min, IQR & $115(90-189)$ & $105(80-133)$ & 0.773 \\
Duration of ACC, min, IQR & $88(51-139)$ & $75(54-100)$ & 0.014 \\
Perioperative transfusion, $\mathrm{n}(\%)$ & $12(75)$ & $63(69)$ & 0.146 \\
Morbidity endpoints, $\mathrm{n}(\%)$ & $10(63)$ & $30(31)$ & 0.032 \\
Hemostatic reoperation, $\mathrm{n}(\%)$ & $2(13)$ & $3(3)$ & 0.223 \\
Acute kidney injury, $\mathrm{n}(\%)$ & $8(50)$ & $22(23)$ & $>0.999$ \\
Prolonged ventilation $>24$ h, n (\%) & $4(25)$ & $11(11)$ & $>0.999$ \\
Stroke, n (\%) & $0(0)$ & $1(1)$ & 0.095 \\
Deep sternal wound infection, n (\%) & $0(0)$ & $2(2)$ & 0.02 \\
In-hospital mortality, n (\%) & $2(13)$ & $2(2)$ & 0.002 \\
Intensive care unit days, IQR & $3(2-7)$ & $2(1-3)$ & $10(8-16)$ \\
Hospital days, IQR & $17(12-27)$ & & \\
\hline
\end{tabular}

Data are presented as mean \pm standard deviation (SD), median (interquartile range, IQR), or number of patients (\%)

$C A B G$ coronary artery bypass grafting, $M A P$ mean arterial pressure, $C V P$ central venous pressure, $C P B$ cardiopulmonary bypass, $I Q R$ interquartile range, $A C C$ aortic cross clamp

\begin{tabular}{|c|c|c|c|c|}
\hline \multirow[t]{2}{*}{ Variables } & \multicolumn{2}{|l|}{ Univariate analysis } & \multicolumn{2}{|l|}{ Multivariate analysis } \\
\hline & Odds ratio $(95 \% \mathrm{CI})$ & $P$ value & Odds ratio $(95 \% \mathrm{CI})$ & $P$ value \\
\hline Pre-operative statin use & $0.179(0.039-0.832)$ & 0.028 & $0.231(0.046-1.155)$ & 0.074 \\
\hline $\begin{array}{l}\text { Pre-operative geriatric } \\
\text { depression scale (short } \\
\text { form) }\end{array}$ & $1.214(1.024-1.439)$ & 0.025 & $1.194(0.987-1.446)$ & 0.069 \\
\hline Baseline $\mathrm{rSO}_{2}<60 \%$ & $5.919(1.584-22.122)$ & 0.008 & $6.748(1.647-27.652)$ & 0.008 \\
\hline Left MCA MFV & $0.986(0.945-1.029)$ & 0.519 & & \\
\hline Right MCA MFV & $0.993(0.954-1.034)$ & 0.735 & & \\
\hline
\end{tabular}

$\mathrm{CI}$ confidence interval, $r \mathrm{SO}_{2}$ relative cerebral oxygen saturation, $M C A M F V$ middle cerebral artery mean blood flow velocity vascular compliance with aging [23]. Nonetheless, MCA MFV could accurately discriminate patients with delirium superimposed on dementia, and a reversible increase in MCA MFV could be observed after resolution of delirium in elderly, non-surgical patients [6]. Even so, MCA MFV is only an indicator of cerebral blood flow, and does not provide a direct measure of metabolic demands. Based on our results, it does not seem to provide information regarding the cerebral perfusion reserve and its predictive role on delirium occurrence is not evident.
In contrast to MCA MFV, a low preoperative $\mathrm{rSO}_{2}$ value $(<60 \%)$ was identified as an independent risk factor for delirium. This result is congruent with the results of previous studies [10,12], in which baseline $\mathrm{rSO}_{2}$ values have been suggested to reflect cognitive reserve $[10,12]$. The cut-off value of preoperative $\mathrm{rSO}_{2}$ value that best predicted delirium was $59.5 \%$ in the present study. This value was comparable to the previously reported cut-off value of $60 \%$ that we used for our analysis [12]. Owing to the suboptimal reserve of cerebral perfusion in patients with a low preoperative $\mathrm{rSO}_{2}$ 


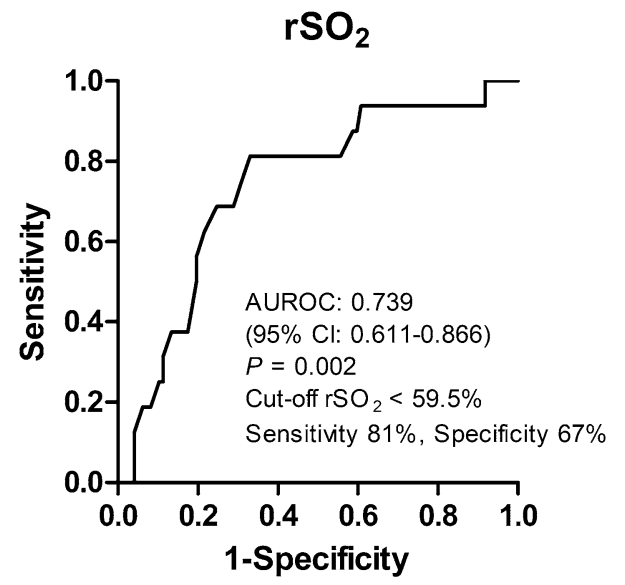

Fig. 3 The receiver operating characteristic curves for baseline $\mathrm{rSO}_{2}$ value on postoperative delirium. $r \mathrm{SO}_{2}$ relative cerebral oxygen saturation, $A U R O C$ area under the receiver operating characteristic, $C I$ confidence interval

value, a small decrease in cerebral perfusion might have led to delirium. In terms of its role as a continuous monitor, monitoring cumulative intraoperative area of decrease in $\mathrm{rSO}_{2}$ value under various cut-off values, and intraoperative interventions to restore $\mathrm{rSO}_{2}$ to an arbitrary target value, have been tested with the goal of detecting or reducing neurocognitive decline in cardiac surgery; however, the results have been inconsistent [10,24].

Of interest, preoperative statin use and SGDS score were shown to be associated with postoperative delirium without statistical significance. The protective effect of statin against the development of delirium was depicted by a large prospective cohort study of cardiac surgical patients [25], which was suggested to be attributable to its endothelial protective action in combination with its anti-inflammatory and immunomodulatory effects [26]. Depressive symptoms have also been identified as risk factors of postoperative delirium in several studies [16, 27]. Cogent mechanisms linking depression with delirium include decreased serotonergic activity in the brain, elevated concentration of cortisol and/or poor physical condition [27].

An interesting observation of the current study is the higher incidence of acute kidney injury in patients who exhibited delirium. A close correlation between these two complications has been implicated previously, which may be attributable to the following reasons. Experimental data indicate that acute kidney injury could lead to inflammation in the brain [28]. Furthermore, kidney dysfunction might reduce the clearance of medications, metabolites, or other potential neurotoxins after cardiac surgery. Although few studies have shown this relationship in patients undergoing cardiac surgery, a study in critically ill patients has shown similar results [29].
The present study is subject to the following limitations. First, the overall delirium incidence was lower than expected (16\% vs. $20 \%$ ), limiting the statistical power. Second, about $30 \%$ of enrolled patients dropped out because of poor acoustic temporal window, and this could have produced a selection bias. This incidence, however, was similar to those reported in other previous studies involving Korean patients, and was accounted for in the sample size calculation when the study was designed [30]. Lastly, the postoperative incidence of acute kidney injury was greater in the delirium group, although its exact temporal relationship with delirium cannot be properly analyzed. However, acute kidney injury as a covariate of delirium has been shown not to alter the relationship between $\mathrm{rSO}_{2}$ and delirium [31].

In conclusion, preoperative MCA MFV measured by TCD failed to provide information regarding the occurrence of delirium after valvular heart surgery in elderly patients, but a low baseline $\mathrm{rSO}_{2}$ value $(<60 \%)$, reflecting inadequacy of the reserve of cerebral perfusion, was associated with a sevenfold increased risk of delirium. Preoperative statin use may be beneficial in the prevention of delirium that merits further studies.

Acknowledgements The current work received exclusive support from departmental resources.

Author contributions Conceptualization: Young-Lan Kwak; Methodology: Young-Lan Kwak, Sarah Soh; Formal analysis and investigation: Sarah Soh, Jong-Wook Song, Nakcheoul Choi; Writing —original draft preparation: Sarah Soh; Writing — review and editing: Jae-Kwang Shim, Young-Lan Kwak; Resources: Jae-Kwang Shim, Young-Lan Kwak; Supervision: Young-Lan Kwak.

Funding The current work received exclusive support from departmental resources.

\section{Compliance with ethical standards}

Conflict of interest The authors declare that they have no conflict of interest.

Ethical approval All procedures performed in studies involving human participants were in accordance with the ethical standards of the institutional and/or national research committee (Institutional Review Board of the Yonsei University Health System, reference number-4-2015-0319) and with the 1964 Helsinki declaration and its later amendments or comparable ethical standards.

\section{References}

1. Saczynski JS, Marcantonio ER, Quach L, Fong TG, Gross A, Inouye SK, Jones RN. Cognitive trajectories after postoperative delirium. N Engl J Med. 2012;367(1):30-9. https://doi. org/10.1056/NEJMoa1112923.

2. Hogue CW Jr, Palin CA, Arrowsmith JE. Cardiopulmonary bypass management and neurologic outcomes: an evidence-based appraisal 
of current practices. Anesth Analg. 2006;103(1):21-37. https://doi. org/10.1213/01.ane.0000220035.82989.79.

3. Steiner LA. Postoperative delirium. Part 1: pathophysiology and risk factors. Eur J Anaesthesiol. 2011;28(9):628-36. https://doi. org/10.1097/eja.0b013e328349b7f5.

4. Caplan GA, Kvelde T, Lai C, Yap SL, Lin C, Hill MA. Cerebrospinal fluid in long-lasting delirium compared with Alzheimer's dementia. J Gerontol Ser A. 2010;65(10):1130-6. https://doi. org/10.1093/gerona/glq090.

5. Sabayan B, Jansen S, Oleksik AM, van Osch MJ, van Buchem MA, van Vliet P, de Craen AJ, Westendorp RG. Cerebrovascular hemodynamics in Alzheimer's disease and vascular dementia: a meta-analysis of transcranial Doppler studies. Ageing Res Rev. 2012;11(2):271-7. https://doi.org/10.1016/j.arr.2011.12.009.

6. Caplan GA, Lan Z, Newton L, Kvelde T, McVeigh C, Hill MA. Transcranial Doppler to measure cerebral blood flow in delirium superimposed on dementia. A cohort study. J Am Med Directors Assoc. 2014;15(5):355-60. https://doi.org/10.1016/j.jamda .2013.12.079.

7. Rudolph JL, Babikian VL, Treanor P, Pochay VE, Wigginton JB, Crittenden MD, Marcantonio ER. Microemboli are not associated with delirium after coronary artery bypass graft surgery. Perfusion. 2009;24(6):409-15. https://doi.org/10.1177/0267659109358207.

8. Zheng F, Sheinberg R, Yee MS, Ono M, Zheng Y, Hogue CW. Cerebral near-infrared spectroscopy monitoring and neurologic outcomes in adult cardiac surgery patients: a systematic review. Anesth Analg. 2013;116(3):663-76. https://doi.org/10.1213/ANE.0b013e3182 $77 \mathrm{a} 255$.

9. Weyland A, Stephan H, Kazmaier S, Weyland W, Schorn B, Grune F, Sonntag H. Flow velocity measurements as an index of cerebral blood flow. Validity of transcranial Doppler sonographic monitoring during cardiac surgery. Anesthesiology. 1994;81(6):1401-10.

10. Lei L, Katznelson R, Fedorko L, Carroll J, Poonawala H, Machina M, Styra R, Rao V, Djaiani G. Cerebral oximetry and postoperative delirium after cardiac surgery: a randomised, controlled trial. Anaesthesia. 2017;72(12):1456-66. https://doi.org/10.1111/anae.14056.

11. Messerotti Benvenuti S, Zanatta P, Longo C, Mazzarolo AP, Palomba D. Preoperative cerebral hypoperfusion in the left, not in the right, hemisphere is associated with cognitive decline after cardiac surgery. Psychosom Med. 2012;74(1):73-80. https://doi. org/10.1097/PSY.0b013e3182383a94.

12. Schoen J, Meyerrose J, Paarmann H, Heringlake M, Hueppe M, Berger KU. Preoperative regional cerebral oxygen saturation is a predictor of postoperative delirium in on-pump cardiac surgery patients: a prospective observational trial. Crit Care (London, England). 2011;15(5):R218. https://doi.org/10.1186/cc10454.

13. Heo EY, Lee BJ, Hahm BJ, Song EH, Lee HA, Yoo CG, Kim YW, Han SK, Shim YS, Lee SM. Translation and validation of the Korean confusion assessment method for the intensive care unit. BMC Psychiatry. 2011;11:94. https://doi.org/10.1186/1471-244x-11-94.

14. Bae JN, Cho MJ. Development of the Korean version of the Geriatric Depression Scale and its short form among elderly psychiatric patients. J Psychosom Res. 2004;57(3):297-305. https://doi. org/10.1016/j.jpsychores.2004.01.004.

15. Janz DR, Abel TW, Jackson JC, Gunther ML, Heckers S, Ely EW. Brain autopsy findings in intensive care unit patients previously suffering from delirium: a pilot study. J Crit Care. 2010;25(3):538. https ://doi.org/10.1016/j.jcrc.2010.05.004.

16. Rudolph JL, Jones RN, Levkoff SE, Rockett C, Inouye SK, Sellke FW, Khuri SF, Lipsitz LA, Ramlawi B, Levitsky S, Marcantonio ER. Derivation and validation of a preoperative prediction rule for delirium after cardiac surgery. Circulation. 2009;119(2):229-36. https://doi.org/10.1161/circulationaha.108.795260.

17. Sorteberg W, Lindegaard KF, Rootwelt K, Dahl A, Russell D, Nyberg-Hansen R, Nornes H. Blood velocity and regional blood flow in defined cerebral artery systems. Acta Neurochir. 1989;97(1-2):47-52.
18. Schramm P, Klein KU, Falkenberg L, Berres M, Closhen D, Werhahn KJ, David M, Werner C, Engelhard K. Impaired cerebrovascular autoregulation in patients with severe sepsis and sepsis-associated delirium. Crit Care (London, England). 2012;16(5):R181. https ://doi.org/10.1186/cc11665.

19. Stump DA, Rogers AT, Hammon JW, Newman SP. Cerebral emboli and cognitive outcome after cardiac surgery. J Cardiothorac Vasc Anesth. 1996;10(1):113-8.

20. Rodriguez RA, Rubens FD, Wozny D, Nathan HJ. Cerebral emboli detected by transcranial Doppler during cardiopulmonary bypass are not correlated with postoperative cognitive deficits. Stroke: J Cereb Circ. 2010;41(10):2229-35. https://doi.org/10.1161/strok eaha.110.590513.

21. Franck M, Nerlich K, Neuner B, Schlattmann P, Brockhaus WR, Spies CD, Radtke FM. No convincing association between postoperative delirium and post-operative cognitive dysfunction: a secondary analysis. Acta Anaesthesiol Scand. 2016;60(10):1404-14. https://doi.org/10.1111/aas.12779.

22. Mufti HN, Hirsch GM. Perioperative prediction of agitated (hyperactive) delirium after cardiac surgery in adults - the development of a practical scorecard. J Crit Care. 2017;42:192-9. https://doi. org/10.1016/j.jcrc.2017.07.045.

23. Yang D, Cabral D, Gaspard EN, Lipton RB, Rundek T, Derby CA. Cerebral hemodynamics in the elderly: a transcranial Doppler study in the Einstein aging study cohort. J Ultrasound Med. 2016;35(9):1907-14. https://doi.org/10.7863/ultra.15.10040.

24. Hong SW, Shim JK, Choi YS, Kim DH, Chang BC, Kwak YL. Prediction of cognitive dysfunction and patients' outcome following valvular heart surgery and the role of cerebral oximetry. Eur J Cardiothorac Surg. 2008;33(4):560-5. https://doi.org/10.1016/j.ejcts .2008.01.012.

25. Katznelson R, Djaiani GN, Borger MA, Friedman Z, Abbey SE, Fedorko L, Karski J, Mitsakakis N, Carroll J, Beattie WS. Preoperative use of statins is associated with reduced early delirium rates after cardiac surgery. Anesthesiology. 2009;110(1):67-73. https:// doi.org/10.1097/ALN.0b013e318190b4d9.

26. Blanco-Colio LM, Tunon J, Martin-Ventura JL, Egido J. Antiinflammatory and immunomodulatory effects of statins. Kidney Int. 2003;63(1):12-23. https://doi.org/10.1046/j.1523-1755.2003.00744 .x.

27. Kazmierski J, Kowman M, Banach M, Pawelczyk T, Okonski P, Iwaszkiewicz A, Zaslonka J, Sobow T, Kloszewska I. Preoperative predictors of delirium after cardiac surgery: a preliminary study. Gen Hosp Psychiatry. 2006;28(6):536-8. https://doi.org/10.1016/j.genho sppsych.2006.08.007.

28. Liu M, Liang Y, Chigurupati S, Lathia JD, Pletnikov M, Sun Z, Crow M, Ross CA, Mattson MP, Rabb H. Acute kidney injury leads to inflammation and functional changes in the brain. J Am Soc Nephrol. 2008;19(7):1360-70. https://doi.org/10.1681/asn.20070 80901.

29. Siew ED, Fissell WH, Tripp CM, Blume JD, Wilson MD, Clark AJ, Vincz AJ, Ely EW, Pandharipande PP, Girard TD. Acute kidney injury as a risk factor for delirium and coma during critical illness. Am J Respir Crit Care Med. 2017;195(12):1597-607. https://doi. org/10.1164/rccm.201603-0476OC.

30. Ahn MK, Lee JS, Hong JM. Feasibility of 1.6-MHz probe for detection of cerebral blood flow in the poor temporal window. J Korean Neurol Assoc. 2014;32(1):8-13.

31. Mailhot T, Cossette S, Lambert J, Cournoyer A, Denault AY. Cerebral oximetry as a biomarker of postoperative delirium in cardiac surgery patients. J Crit Care. 2016;34:17-23. https://doi. org/10.1016/j.jcrc.2016.02.024.

Publisher's Note Springer Nature remains neutral with regard to jurisdictional claims in published maps and institutional affiliations. 